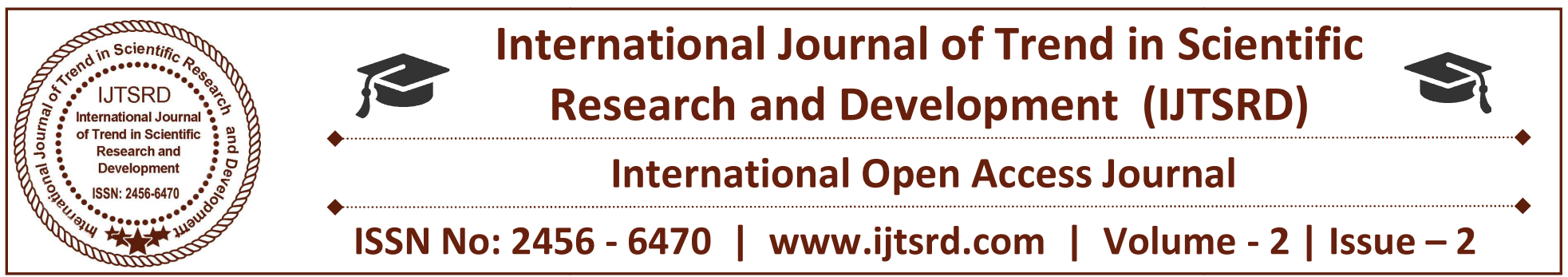

\title{
Smart Bag with Theft Prevention and Real Time Tracking
}

\author{
Ankush Sutar, Tukaram Kocharekar, Piyush Mestry, Prathamesh Sawantdesai, Mrs. Suhasini S. Goilkar \\ Department of EXTC, Finolex academy of Management \& Technology, \\ Ratnagiri, Maharashtra, India
}

\begin{abstract}
In our day to day life travelling has become one of the important aspects for human being. Generally for travelling purpose people uses normal luggage bags or suitcase but in today's world such types of bags are not safe from the security point of view and less hard work and comfort of having better journey. Nowadays field of electronic has designed with advanced electronic technology that can provide facilities like surveillance. All the electronic inventions are to reduce manual effort upon mechanical work and to create an interaction between human and machine. Human following bags are one of the finest technologies in electronics and by utilizing its advantages and applications in day to day life in this paper we have tried to mention all the study related to smart bag. Several techniques are introduced to follow bag behind the owner for the following features human detection done by ultrasonic sensors. In terms of privacy the bag can be activated by owner's identity and also location can be tracked using GPS and GSM. In this bag within a small platform all the facilities are implemented together efficiently.
\end{abstract}

Keywords: GPS, GSM, Bluetooth, Arduino, UV sensor

\section{INTRODUCTION}

Smart bag is designed in such a way that it is light weight luggage bag which is modified with advanced electronic technology for the purpose of advanced security system and also made the human travelling facilities more efficient with less effort. Auto trailing technology which reduces human efforts. Wherever the people travel they used to carry luggage especially to airport all of them dragging out their heavy luggage perhaps trailing of the bag is very difficult task for old peoples. If bag that follows passengers by utilizing human following concept then entire problem get vanished. Following technique is implemented using data taken from ultrasonic and IR sensor. Ultrasonic sensor always measure distance between bag and human by sending sound waves and collects the reflected waves when it tracks an obstacle. Misplace or loosing of bag is also avoidable using proximity detection method. Beyond this it has feature of tracing and tracking the bag using GPS and GSM and locate the accurate position of the bag. Fingerprint locking system is used in this project Recharging port is also provided in this project. For recharging port an in built power bank is used. Recharging port mainly used for charging of mobiles phones and laptops.

\section{LITERATURE SURVEY}

\section{1) Design and Implementation of Smart Bag}

This paper suggests us about design and construction of smart bag. Human following activity can be done by using two methods first by using UV and IR sensors and second by using web camera. We are going to use UV and IR sensors to perform this task [1]. Security is most important factor in daily life so providing protection to bag is necessary. Ensuring safety of peoples and their valuable things is very important for the prevention of illegal handling. The ability to track and trace from anywhere on the earth has been mankind's unfulfilled desire. GPS and GSM are used to get the exact location of the bag. 
Fingerprint locking system provides advanced security to the bag. This project is actually an interfacing of small mini projects in order to implement the whole project.

\section{BLOCK DIAGRAM}

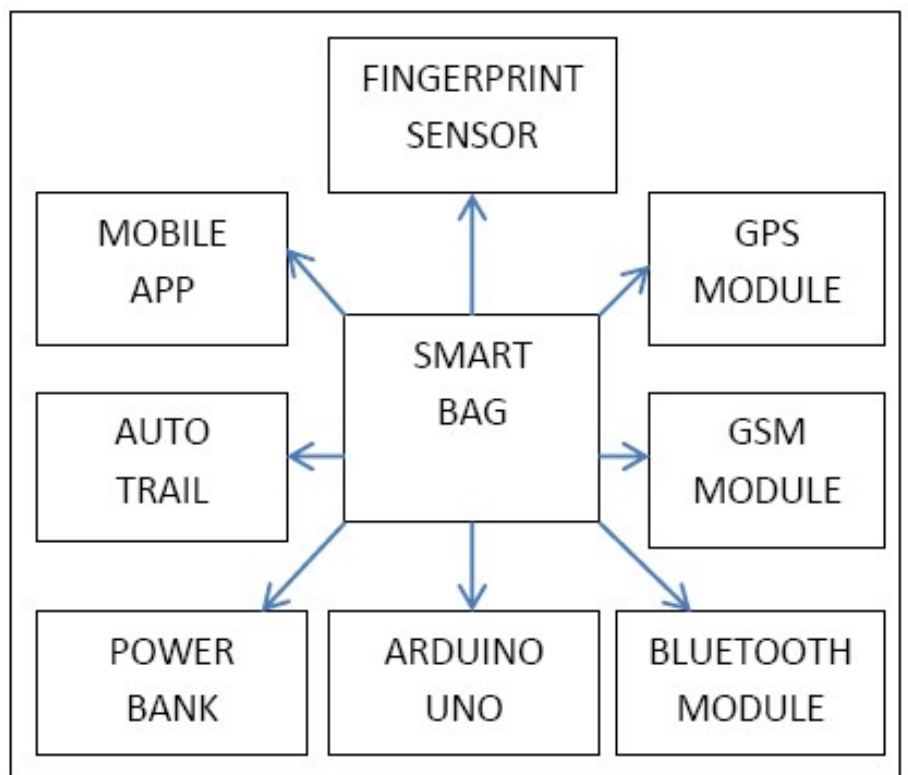

Fig 1: Block diagram of the smart bag

\section{2) GSM and GPS used for Tracking and Tracing of Smart Bag}

Tracking systems were first developed for the shipping industry to track cargo. First devices developed were passive. To obtain automatic and real time tracking active devices are to be used. Muruganandham and P. R. Mukesh proposed a system that uses GSM/GPRS modem and GPS system to provide real time tracking over the internet by TCP/IP connection through Java applications developed specifically for it. External databases are used to maintain the tracking details. The tracking device consists of the GPS, GSM modem and the Arduino. Location name and GPS coordinate values are stored as a LUT (Look up Table) in the Arduino. As soon as the GSM modem receives SMS request for location, the microcontroller checks for a closest location match inside the LUT with the received GPS coordinate data. The matched location detail is sent to the user as an SMS using GSM modem thus completing the request. Further service enhancements like breakdown alert can be made into the proposed system in a cost effective manner. [2]

\section{3) Fingerprint locking system}

Fingerprint recognition technology allows access to only those whose fingerprints that are pre stored in the memory. Stored fingerprints are retained even in the event of complete power failure or battery drain. These eliminates the need for keeping track of keys or remembering a combination password, or PIN. It can only be opened when an authorized user is present, since there are no keys or combinations to be copied or stolen, or locks that can be picked. The fingerprint based lock therefore provides a wonderful solution to conventionally encountered inconveniences. This report focuses on the use of fingerprints to unlock locks, as opposed to the established method of using keys. Fingerprints are patterns of ridges and valleys on the surface of the finger. Like everything in the human body, these ridges form through a combination of genetic and environmental factors. The genetic code in DNA gives general orders on the way skin should form in a developing fetus, but the specific way it forms is a result of random events. [3]

\section{4) Auto Trailing Bag of the Smart Bag}

\section{a) Object detection using ultrasonic sensors:}

The bag can be activated by sending an SMS from the mobile device. Two ultrasonic sensors are used to detect human presence and are connected in such a way that the transmitter section of each sensor is placed in middle and receiver section is placed on two sides. The transmitter section continuously emits sound waves through air and would return when it strikes the obstacles on the way. The forward, right and left movement of the bag is based on the signal strength received at each receiver section. The algorithm compares the readings from the two sensors and decides whether to turn left (if the left sensor returns maximum signal), right or to forward (if the output of the two sensors is similar). If the range drops or the distance between the bag and human is less than a predefined value, the bag stops.

\section{b) Human following mechanism using two IR sensors.}

The two IR sensors are used to perform the following mechanism. On one of the side of bag two receivers are placed at both the ends and one transmitter is placed at the Centre. So whenever person turns left or rights the action is detected by the two IR receivers placed at the corners of the bag. And the data taken by 
the IR receiver is given to the arduino so by performing programming the motors are rotated in the particular direction so as to follow the person [4].

\section{5) Proximity detection using Bluetooth module}

Proximity detection is used to keep yours luggage close. If someone unknowingly took your bag and is strayed outside the range of your bluetooth signal then alert message will display on your mobile app. Then using real time GPS we can track the bag. The connecting distance is fixed between the bag and mobile say 10 meters [5].

\section{CONCLUSION}

Smart bag is an innovative carry on suitcase that makes life easier and smoother. Carrying luggage is the main difficulty faced by each and every passenger. Here we try to solve the dragging of luggage difficulty and also providing better security and intelligent features that suitable for modern era. In this project we developed a new low cost human following technology to assist low cost consumer product implementation, so that the overall production cost of an automatic user following bag will be less. The inbuilt power bank can provide sufficient power and at the same time share power to users gadgets like smartphone, laptops etc.

\section{REFERENCE}

1) E. A. Topp and H. I. Christensen, "Tracking for following and passing persons," in Proc. IEEE/RSJ Int. Conf. Intell. Robots Syst., Edmonton, AB, Canada, 2005, pp. 2321-2327.

2) Muruganandham and P. R. Mukesh (2010) "Real time Web based vehicle tracking using GPS" World academy of science, Engineering and Technology.

3) Koichi I., Ayumi M., Takafumi A., Hiroshi N., Koji Kobayashi, and Tatsuo H. (2005) "A Fingerprint Recognition Algorithm Combining Phase-Based Image Matching and FeatureBasedMatching", http://www.aoki.ecei.tohoku.ac.jp/ ito/cr2114.pdf $-00$

4) Keerthi .S. Nair, Anu Babu Joseph, Jinu Isaac Kuruvilla "Design of a low cost human following porter robot at airports" IJACTE, ISSN (Print): 2319-2526, Volume -3, Issue -2, 2014.

5) Heidi monson (1199) Bluetooth technology and implementation john wiley \& sons. 